\title{
Effect of tai chi versus aerobic exercise for fibromyalgia: comparative effectiveness randomized controlled trial
}

\author{
Chenchen Wang, ${ }^{1}$ Christopher H Schmid, ${ }^{2}$ Roger A Fielding, ${ }^{3}$ William F Harvey, ${ }^{1}$ Kieran F Reid, ${ }^{3}$ \\ Lori Lyn Price, ${ }^{4}$ Jeffrey B Driban, ${ }^{1}$ Robert Kalish, ${ }^{5}$ Ramel Rones, ${ }^{6}$ Timothy McAlindon ${ }^{1}$
}

${ }^{1}$ Center for Complementary and Integrative Medicine and Division of Rheumatology, Tufts Medical Center, Tufts University School of Medicine, Boston, MA 02111, USA

${ }^{2}$ Department of Biostatistics and Center for Evidence

Synthesis in Health, Brown

University School of Public Health, Providence, RI, USA

${ }^{3}$ Nutrition, Exercise Physiology and Sarcopenia Laboratory,

Jean Mayer USDA Human

Nutrition Research Center on

Aging, Tufts University, Tufts

University School of Medicine,

Boston, USA

${ }^{4}$ The Institute for Clinical

Research and Health Policy

Studies, Tufts Medical Center,

Tufts Clinical and Translational

Science Institute, Tufts

University, Boston, MA, USA

${ }^{5}$ Division of Rheumatology, Tufts Medical Center, Tufts University

School of Medicine, Boston,

MA, USA

${ }^{6}$ Center for Mind-Body

Therapies, Boston, MA, USA

Correspondence to: $C$ Wang

cwang2@tuftsmedicalcenter.org

Additional material is published online only. To view please visit

the journal online.

Cite this as: $B M J$ 2018;360:k851 http://dx.doi.org/10.1136/bmj.k851

Accepted: 13 February 2018

\section{ABSTRACT}

OBJECTIVES

To determine the effectiveness of tai chi interventions compared with aerobic exercise, a current core standard treatment in patients with fibromyalgia, and to test whether the effectiveness of tai chi depends on its dosage or duration.

DESIGN

Prospective, randomized, 52 week, single blind comparative effectiveness trial.

\section{SETTING}

Urban tertiary care academic hospital in the United States between March 2012 and September 2016.

\section{PARTICIPANTS}

226 adults with fibromyalgia (as defined by the American College of Rheumatology 1990 and 2010 criteria) were included in the intention to treat analyses: 151 were assigned to one of four tai chi groups and 75 to an aerobic exercise group.

\section{INTERVENTIONS}

Participants were randomly assigned to either supervised aerobic exercise (24 weeks, twice weekly) or one of four classic Yang style supervised tai chi interventions (12 or 24 weeks, once or twice weekly). Participants were followed for 52 weeks. Adherence was rigorously encouraged in person and by telephone.

\section{MAIN OUTCOME MEASURES}

The primary outcome was change in the revised fibromyalgia impact questionnaire (FIQR) scores at 24 weeks compared with baseline. Secondary outcomes included changes of scores in patient's global assessment, anxiety, depression, self efficacy, coping

\section{WHAT IS ALREADY KNOWN ON THIS TOPIC}

Emerging evidence suggests that tai chi mind-body practice has short term therapeutic benefits for chronic musculoskeletal pain and mental health conditions

Three randomized trials and three non-randomized, single arm studies found that tai chi alleviates pain and improves physical and mental health in patients with fibromyalgia but concluded that larger and more rigorous trials are needed to confirm the results

Whether tai chi is better than aerobic exercise, a currently recommended core of standard care, and what the ideal dose (frequency or duration) for treating fibromyalgia is remain unknown

\section{WHAT THIS STUDY ADDS}

This study suggests that tai chi mind-body treatment for fibromyalgia has similar or greater benefits than standard care treatment

A longer duration of tai chi had more benefit than a shorter duration

The therapeutic benefits of tai chi were consistent among three instructors in a large sample of diverse patients with fibromyalgia, which provide more support for generalizability to other settings and patient populations

strategies, physical functional performance, functional limitation, sleep, and health related quality of life.

\section{RESULTS}

FIQR scores improved in all five treatment groups, but the combined tai chi groups improved statistically significantly more than the aerobic exercise group in FIQR scores at 24 weeks (difference between groups $=5.5$ points, $95 \%$ confidence interval 0.6 to $10.4, P=0.03$ ) and several secondary outcomes (patient's global assessment $=0.9$ points, 0.3 to 1.4 , $\mathrm{P}=0.005$; anxiety $=1.2$ points, 0.3 to $2.1, \mathrm{P}=0.006$; self efficacy $=1.0$ points, 0.5 to $1.6, P=0.0004$; and coping strategies, 2.6 points, 0.8 to $4.3, \mathrm{P}=0.005$ ). Tai chi treatment compared with aerobic exercise administered with the same intensity and duration (24 weeks, twice weekly) had greater benefit (between group difference in FIQR scores $=16.2$ points, 8.7 to 23.6, P<0.001). The groups who received tai chi for 24 weeks showed greater improvements than those who received it for 12 weeks (difference in FIQR scores=9.6 points, 2.6 to $16.6, \mathrm{P}=0.007$ ). There was no significant increase in benefit for groups who received tai chi twice weekly compared with once weekly. Participants attended the tai chi training sessions more often than participants attended aerobic exercise. The effects of tai chi were consistent across all instructors. No serious adverse events related to the interventions were reported.

\section{CONCLUSION}

Tai chi mind-body treatment results in similar or greater improvement in symptoms than aerobic exercise, the current most commonly prescribed nondrug treatment, for a variety of outcomes for patients with fibromyalgia. Longer duration of tai chi showed greater improvement. This mind-body approach may be considered a therapeutic option in the multidisciplinary management of fibromyalgia.

\section{TRIAL REGISTRATION \\ ClinicalTrials.gov NCT01420640.}

\section{Introduction}

Fibromyalgia is a complex disorder characterized by chronic widespread musculoskeletal pain, fatigue, sleep disturbance, and prominent physical and psychological impairment, causing substantial and increasing healthcare costs. ${ }^{1}$ It affects approximately $2-4 \%$ of the general population between 18 and 65 years of age worldwide. ${ }^{2}$ The prevailing causal paradigm views it as a disorder of pain regulation associated with neuroendocrinologic changes in the central and peripheral nervous systems. ${ }^{3}$ Although there is no cure for fibromyalgia, core treatments combine multidisciplinary approaches including 
drugs, exercise, cognitive behavioral therapy, and health education.

Drug interventions such as analgesics seem to confer modest short term benefits for fibromyalgia symptoms, but recent reviews question whether they have a sustained, clinically meaningful response..$^{4-6}$ Many patients discontinue their therapies owing to lack of efficacy, toxicity, or intolerance to the drug. Additionally, $11 \%$ to $69 \%$ of patients in recent large retrospective database studies reported use of short acting or long acting opioids, which carries the risk of dependency and misuse, ${ }^{7}$ despite evidence showing that patients with fibromyalgia receiving opioids have poorer health outcomes than those receiving nonopioids. ${ }^{8}$ Evidently, pharmacotherapy is insufficient to resolve persistent symptoms and improve quality of life for patients with fibromyalgia.

Over the past decades, the benefits of exercise training have been documented in the literature, ${ }^{9-16}$ and moderate aerobic exercise is currently recommended as part of standard care for the management of fibromyalgia. ${ }^{17-19}$ However, many patients have difficulties performing and adhering to exercise programs owing to fluctuations in symptoms, and they remain unfit. $^{12-15}$ New approaches are needed that patients can embrace to reduce chronic musculoskeletal pain and improve their physical and psychological functioning and quality of life.

Tai chi is an ancient discipline involving exercise rooted in traditional Chinese medicine that originated as a martial art and has been practiced for many centuries. This complex, multicomponent mindbody intervention integrates physical, psychosocial, spiritual, and behavioral elements to promote health and fitness. ${ }^{2021}$ Two previous 12 week randomized trials found that tai chi effectively alleviates pain and improves physical and mental health in patients with fibromyalgia compared with wellness education or stretching controls. ${ }^{22}{ }^{23}$ Similar beneficial effects of tai chi have been shown in patients with osteoarthritis and rheumatoid arthritis. ${ }^{24-27}$ Despite evidence suggesting that tai chi has therapeutic benefits for musculoskeletal conditions, the relative benefits and harms of tai chi compared with aerobic exercise, a common treatment for this population, are unknown. Furthermore, the ideal dose (frequency or duration) of tai chi as a treatment for fibromyalgia has not been determined.

We compared the effectiveness of tai chi versus aerobic exercise in a large population with fibromyalgia symptoms followed for 12 months and tested whether the effectiveness of tai chi depends on its dosage and duration. We hypothesized that participants receiving tai chi compared with aerobic exercise would have greater improvements in fibromyalgia symptom severity as well as in physical and psychosocial functioning and quality of life; that participants receiving a longer duration (24 weeks) of tai chi would show greater improvements than those receiving a shorter duration (12 weeks); and that participants receiving a higher frequency of tai chi (twice weekly) would show greater improvements than those receiving a lower frequency (once weekly).

\section{Methods}

This was a randomized, 52 week, single blind comparative effectiveness trial. Participants with fibromyalgia were randomly assigned to either supervised aerobic exercise twice weekly for 24 weeks or to one of four tai chi interventions: 12 or 24 weeks of supervised tai chi completed once or twice weekly. We followed participants for 52 weeks. The primary outcome was the change in revised fibromyalgia impact questionnaire (FIQR) total score from baseline to 24 weeks. ${ }^{28}$ Details of the trial design and conduct are published elsewhere. ${ }^{29}$

\section{Setting and participants}

The trial was conducted at Tufts Medical Center, a tertiary care academic hospital in Boston, Massachusetts. Patients were recruited through a combination of advertisements and enrollment through clinics in the greater Boston area. Prescreening was administered by telephone before scheduling a visit onsite involving clinical examinations according to the standardized protocol. Participants provided written informed consent before baseline assessments for eligibility. Self reported race/ethnicity and sex were also collected. We enrolled eligible participants who were 21 years or older and fulfilled the American College of Rheumatology 1990 and 2010 preliminary diagnostic criteria for fibromyalgia. ${ }^{30} 31$ These criteria include a history of widespread bilateral musculoskeletal pain both above and below the waist for a minimum of three months and pain in at least 11 of 18 specific tender points, with moderate or greater tenderness reported on digital palpation. ${ }^{30}$ Participants needed to have a widespread pain index of 7 or more and symptom severity scale score of 5 or more, or widespread pain index of 3-6 and symptom severity scale score of 9 or more, and not have a disorder that would otherwise explain the pain. ${ }^{31}$ Participants also had to be willing to complete the 12 week or 24 week intervention, including exercise sessions once or twice weekly. We excluded those who had participated in tai chi or other similar types of complementary and alternative medicine within the past six months; those with serious medical conditions that might limit their participation; those with other diagnosed medical conditions, such as inflammatory arthritis or connective tissue diseases; women who were pregnant or were planning a pregnancy during the study period; those who did not speak English; and those who were unable to pass the mini-mental state examination (score $<24$ out of 30). ${ }^{32}$ Participants were able to continue routine drugs and usual visits to their physicians throughout the study.

\section{Randomization and masking}

After the study rheumatologists (WFH or RK) confirmed that participants met the eligibility criteria, baseline assessments were performed. In the three 
weeks before the start of each of six enrollment cycles, we completed baseline assessments for a group of 40 to 50 prescreened participants to obtain an eligible cohort to randomize. Randomization occurred after the baseline evaluation in six consecutive enrollment cycles. The study statistician (CHS) generated random sequences in the $\mathrm{R}$ statistical package, blocking on instructor within the tai chi group and unblocked within the aerobic exercise group. We generated a new random sequence for each cycle so that it was also blocked on cycle. Each enrollment cycle consisted of an aerobic exercise intervention group and two of the four tai chi intervention groups. The tai chi intervention groups were rotated through the six cycles so that each treatment regimen (12 or 24 weeks, once or twice weekly) occurred three times over the course of the study. We used a factorial arrangement to ensure that each of the three tai chi instructors conducted each of the four treatments once throughout the trial. This design allowed for assessment of instructor level effects in the tai chi group. Assignments were concealed in sealed, opaque envelopes and were only opened for each participant after the study coordinator obtained consent and confirmed eligibility.

The research study nurses, physical function assessors, and sponsor staff who conducted the baseline and follow-up assessments were blinded to treatment allocation.

\section{Interventions}

A detailed description of the classic Yang style tai chi and aerobic exercise protocol has been published elsewhere. ${ }^{29}$ The tai chi and aerobic exercise groups ran concurrently to remove potential confounding from seasonal influences on symptom severity. The five groups received educational information about the importance of physical activity and home practice. Each tai chi session lasted 60 minutes, once or twice a week, for 12 weeks or 24 weeks. Aerobic exercise sessions were 60 minutes, twice a week, for 24 weeks. Participants were encouraged to integrate at least 30 minutes of tai chi or aerobic exercise into their daily routine during their interventions. They were also asked to continue exercise after completing their 12 week or 24 week sessions, as well as throughout 52 weeks of follow-up. Attendance forms and sign-in sheets were used to monitor attendance of each participant at treatment sessions. Study staff conducted monthly calls to encourage adherence to home practice throughout 52 weeks of follow-up. Adherence to the protocol was defined as the proportion of study sessions attended.

Tai chi

Before study initiation, we developed a standardized classic Yang style tai chi protocol for fibromyalgia based on the literature. ${ }^{33}$ Three experienced instructors were recruited from the greater Boston area and attended a training session to review the tai chi protocol ${ }^{29}$ and concepts of fibromyalgia. We monitored all study sessions using video recordings and provided feedback to instructors throughout the study to continuously monitor the quality of the intervention. Participants received printed materials on tai chi principles, techniques, and safety precautions for fibromyalgia. In the first session, the tai chi instructor explained the exercise theory and procedures. The remaining sessions included warm-up and a review of tai chi principles, meditative movements, breathing techniques, and various relaxation methods. Instructors reminded participants in class to maintain their practice daily according to the weekly assignments.

\section{Aerobic exercise}

Participants randomized to aerobic exercise received a closely supervised program in group format cardiovascular exercise consistent with current recommended guidelines of moderate intensity exercises for fibromyalgia. ${ }^{17-19}$ The training was designed to increase heart rate and rate of perceived exertion, thereby improving cardiovascular fitness. ${ }^{34}$ Each aerobic exercise session consisted of several components: an active warm-up including low intensity movements and dynamic stretching; choreographed aerobic training, progressing gradually from low to moderate intensity; and a cool-down involving low intensity movements, and dynamic and static stretching. During all components of the sessions, the instructor monitored accuracy of exercise technique to ensure participant comfort and safety as well as to minimize adverse events. Heart rate and rate of perceived exertion were recorded during each session to monitor exercise intensity. ${ }^{35}$ Two exercise physiology scientists (RAF, KFR) oversaw the program and conducted training sessions for the instructors.

During the first week, participants completed a 15 minute warm-up, 20 minutes of aerobic training (50-60\% of estimated maximum heart rate: rate of perceived exertion 11-13 (from 6 (no exertion) to 20 (maximal exertion)), ${ }^{35}$ and a 25 minute cool-down. We also provided printed materials on exercise principles, practicing techniques, and safety precautions, and explained exercise theory and procedures for fibromyalgia. In subsequent sessions, all participants progressively increased the duration and intensity of exercise through increasing aerobic activity by five minutes every two weeks. In the fibromyalgia population, lower levels of cardiovascular fitness and lower thresholds for post-exercise muscle pain and fatigue are reported. ${ }^{3637}$ Our program was individually tailored to each participant, closely supervised, and introduced in a progressive manner to accommodate these differences. By weeks 10 to 12 the session included 40 minutes of aerobic training (60-70\% of estimated maximum heart rate). We also instructed participants to walk daily, gradually increasing the time until they reached 30 minutes a day. Throughout all sessions, instructors monitored techniques to ensure participant comfort and safety and to minimize adverse events.

\section{Outcome measures}

We assessed key clinical outcomes focused on symptom severity and body pain drawn from previous randomized 
trials, exercise literature, and recommendations by the American College of Rheumatology. ${ }^{9-12} 16222331$ We also evaluated secondary physical, psychological, and psychosocial aspects of fibromyalgia that are well documented in clinical care and research. ${ }^{38}$ Each participant was evaluated at baseline, and at 12, 24, and 52 weeks.

\section{Primary outcome}

The primary outcome was the change in revised FIQR total score (range 0-100, with higher scores indicating greater impact of symptoms) from baseline to the 24 week visit. ${ }^{28}$ The FIQR is a well validated multidimensional instrument that measures participant rated overall severity of fibromyalgia, including intensity of pain, physical function, fatigue, morning tiredness, depression, anxiety, job difficulty, and overall wellbeing. ${ }^{39}$ Each item is standardized on a scale ranging from 0 to 10 , with lower scores indicating more improvement or less negative impact. FIQR was also assessed weekly during the intervention period for all groups of participants. A minimal clinically important difference in the FIQR score has not yet been established. However, the FIQR is an updated version of the original fibromyalgia impact questionnaire (FIQ) with which FIQR scores correlate closely $(r=0.88$, $\mathrm{P}<0.001) .{ }^{39}$ Therefore, we estimated the minimal clinically important difference of the FIQR score based on the established minimal clinically important difference of the FIQ score ( $14 \%$ or 8.1 units). ${ }^{40}$

\section{Secondary outcomes}

Secondary outcomes were measured at baseline and at 12, 24, and 52 weeks and included the patient's global assessment using a visual analog scale (range 0-10, with higher scores indicating greater impact of symptoms); the hospital anxiety and depression scale (HADS, range 0-21, with higher scores indicating more severe symptoms) ${ }^{41}$; the arthritis self efficacy scale (range 1-10, with higher scores indicating greater self efficacy for management of fibromyalgia pain $)^{42}$; the Pittsburgh sleep quality index, the most widely used general measure that has been validated in patients with fibromyalgia (range 0-21, with higher scores indicating worse sleep quality) ${ }^{43}$ (we did not administer the sleep quality numeric rating scale that was also originally proposed); summary scores for the physical and mental components of the short form health survey (range 0-100, with higher scores indicating better health status) ${ }^{44}$; symptom severity scale score (range $0-12$, with higher scores indicating greater severity) ${ }^{31}$; Beck depression inventory II (range 0-63, with higher scores indicating greater depressive symptoms) ${ }^{45}$; coping strategies score (range 0-36, with higher scores indicating better coping ${ }^{46}$; social support survey (range 0-5, with higher scores indicating more social support ${ }^{47}$; improved health assessment questionnaire (range 0-100, with higher scores indicating more disability) ${ }^{48}$; outcome expectations scale (range 1-5, with higher scores indicating higher outcome expectations) ${ }^{49}$; the community health activities model program for seniors (CHAMPS) physical activity questionnaire for older adults ${ }^{50}$; and health assessment questionnaire. ${ }^{4851-54}$ Assessments of physical function included the chair stand, six minute walk, and balance tests. ${ }^{55-58}$ Participants' muscle strength and power were also assessed using a leg press. ${ }^{59}$ Several secondary outcomes will be reported in separate publications. The health assessment questionnaire will be used in an economic evaluation. Muscle strength and power, chair stand, and balance along with CHAMPS will be used in an analysis of the role of muscle and physical function in fibromyalgia.

The research staff, unaware of the participant's group assignment, evaluated physical performance by measuring the distance completed (in meters) in the six minute walk test. ${ }^{60}$ Additional measures included body mass index, outcome expectations for treatment, adherence, and safety.

Monitoring of adverse events and safety procedures Throughout the intervention period, we systematically ascertained adverse events at each contact with the patient and a study rheumatologist (WFH) used a standardised reporting system to evaluate these for relevance to the intervention and severity.

We defined serious adverse events as those that were life threatening, were permanently disabling, were severely incapacitating, required prolonged inpatient hospital stay, or were fatal. In accordance with guidelines we reported adverse events to the human research committee.

\section{Drug use}

Participants maintained their regular drug treatments. We did not change or recommend changes in medical therapy. At each evaluation we recorded any change in use of analgesics, antidepressants, anticonvulsants, muscle relaxants, benzodiazepines, dopamine agonists, and 5 hydroxytryptamine receptor agonists throughout the entire intervention and evaluation period.

\section{Statistical analysis}

Sample size calculations were based on the results of two previous fibromyalgia trials. The first study compared an aerobic and flexibility exercise program with education and detected a mean improvement of 7.9 points in FIQ score, yielding an effect size of $0.62{ }^{10}$ The second compared 12 weeks of tai chi with an attention control and found a mean improvement of 18.4 points in FIQ score for the tai chi group, yielding an effect size of $1.04 .^{22}$ We hypothesized that participants receiving tai chi would improve more than those receiving aerobic exercise. Using a two sided hypothesis test at 0.05 significance level with an allocation ratio of $2: 1$, we determined that a sample size of 216 (144 in tai chi group, 72 in aerobic exercise group) gives approximately $80 \%$ power to detect an effect size difference of 0.4 . In addition, we sought to elucidate how four different dose and frequency combinations might modify the impact of tai chi. By 
assigning 36 participants to each group, we had 80\% power to detect an effect size of 0.67 between two of these groups with a two sided 0.05 level test.

We used longitudinal fixed effects models based on the intent-to-treat principle to determine the comparative efficacy of the five treatments at baseline and at 12, 24, and 52 weeks. We calculated treatment effects for change from baseline to 12, 24, and 52 weeks focusing on predefined comparisons: aerobic exercise versus average of four tai chi groups, average of 12 week versus 24 week tai chi, and average of once weekly versus twice weekly tai chi. In addition, we compared tai chi twice weekly for 24 weeks with aerobic exercise as an exploratory analysis. We considered time and treatment as categorical fixed factors and used an unstructured covariance matrix. The interaction of time and each comparison tested the difference between treatment groups. The primary outcome, change from baseline to 24 weeks, was assessed using the appropriate comparison from the longitudinal model. This protects the inference from data, the missingness of which is related to reported outcomes. As planned, we also examined whether results differed among tai chi instructors through an interaction of instructor with time and treatment in a model fit only to the data from the participants who undertook tai chi. We used a generalized linear mixed model with random intercepts and fixed treatment effects for binary outcomes.

Although the use of the longitudinal model protects against the effects of outcomes missing at random, it does so only if the hypothesized model is correct. Thus for sensitivity analysis, we assessed model assumptions with standard regression diagnostic evaluations ${ }^{61}$ and also examined the association of missingness with baseline predictors and longitudinal outcomes. We conducted several sensitivity analyses, including multiple imputation and adjustment for attendance rate and variables that were found to differ among participants who missed visits. Missing data were multiply imputed using a set of baseline characteristics as well as the 12, 24, and 52 week outcomes. We also assessed the impact of attendance by analyzing treatment effects in the subgroup of participants who attended at least half of the treatment sessions. Results are presented as differences between groups with 95\% confidence intervals based on estimates from the longitudinal models. We considered a two sided $P$ value $<0.05$ to indicate statistical significance. All analyses were performed with SAS version 9.4 (SAS Institute) and R 3.2.

\section{Patient involvement}

Patients were not involved in the development of plans for recruitment, design, outcome measures, or implementation of the study conduct. No patients were asked to advise on the interpretation or writing of results. The burden of the intervention was not assessed, but we assessed outcome expectations for the study interventions. We intend to disseminate the main results to trial participants and will pursue patient and public involvement in the development of an appropriate method of dissemination.

\section{Results}

Between March 2012 and September 2014 we screened 867 potential participants by telephone. Of the 272 who qualified for baseline evaluation, 226 consented, met the eligibility criteria, and were randomized. The remaining 46 participants were excluded for various reasons (fig 1).

Table 1 shows baseline data for the 226 participants. Characteristics were balanced among all groups. The mean age of participants was 52 years, 92\% were women, the racial/ethnic composition was diverse (61\% white), and mean body mass index was $30 \mathrm{~kg} / \mathrm{m}^{2}$. The average duration of body pain was nine years. The average SF-36 physical component score was about two standard deviations below the norm for the general US population, ${ }^{44}$ indicating a cohort with poor health status. Treatment groups did not statistically significantly differ in baseline expectations of benefits from their randomly assigned regimen (outcome expectations scale score: tai chi, 3.8 (SD 0.6); aerobic exercise, 3.9 (SD 0.6)). ${ }^{53}$ Participants assigned to tai chi attended $62 \%$ of possible classes, and participants assigned to aerobic exercise attended $40 \%$ (see appendix table 1). Overall, 183 (81\%) participants completed the 12 week evaluation, 181 (80\%) completed the 24 week evaluation, and 158 (70\%) completed the 52 week evaluation (fig 1).

Table 2 shows changes from baseline to 12, 24, and 52 weeks for the five treatment groups for all continuous outcomes. Table 3 shows differences between groups at all evaluation visits for the main treatment comparisons.

Compared with baseline, FIQR scores improved for participants in all five treatment groups at each follow-up. Generally, participants who undertook tai chi improved at all times on other outcomes as well, whereas participants who undertook aerobic exercise showed less improvement.

At 24 weeks the combined tai chi groups improved significantly more than the aerobic exercise group in FIQR scores (difference between groups $=5.5$ points, $95 \%$ confidence interval 0.6 to $10.4, \mathrm{P}=0.03$ ). Based on the estimated minimal clinically important difference for FIQR score of 8.1 points, ${ }^{40}$ the difference of 5.5 points between the mean scores in the aerobic exercise group and combined tai chi groups at 24 weeks was not clinically important. However, when tai chi was compared with aerobic exercise of matched intensity-that is, twice weekly for 24 weeks, we found that the lower bound of the $95 \%$ confidence interval for the difference between groups exceeded the specified threshold for clinically significant improvement (estimate 16.2 points, 95\% confidence interval 8.7 to $23.6, \mathrm{P}<0.001)$. The lower bound of the $95 \%$ confidence interval of improvement for both 24 week tai chi groups exceeded the minimal clinically important difference as well. In addition, all groups (tai chi and aerobic exercise) improved by more than 


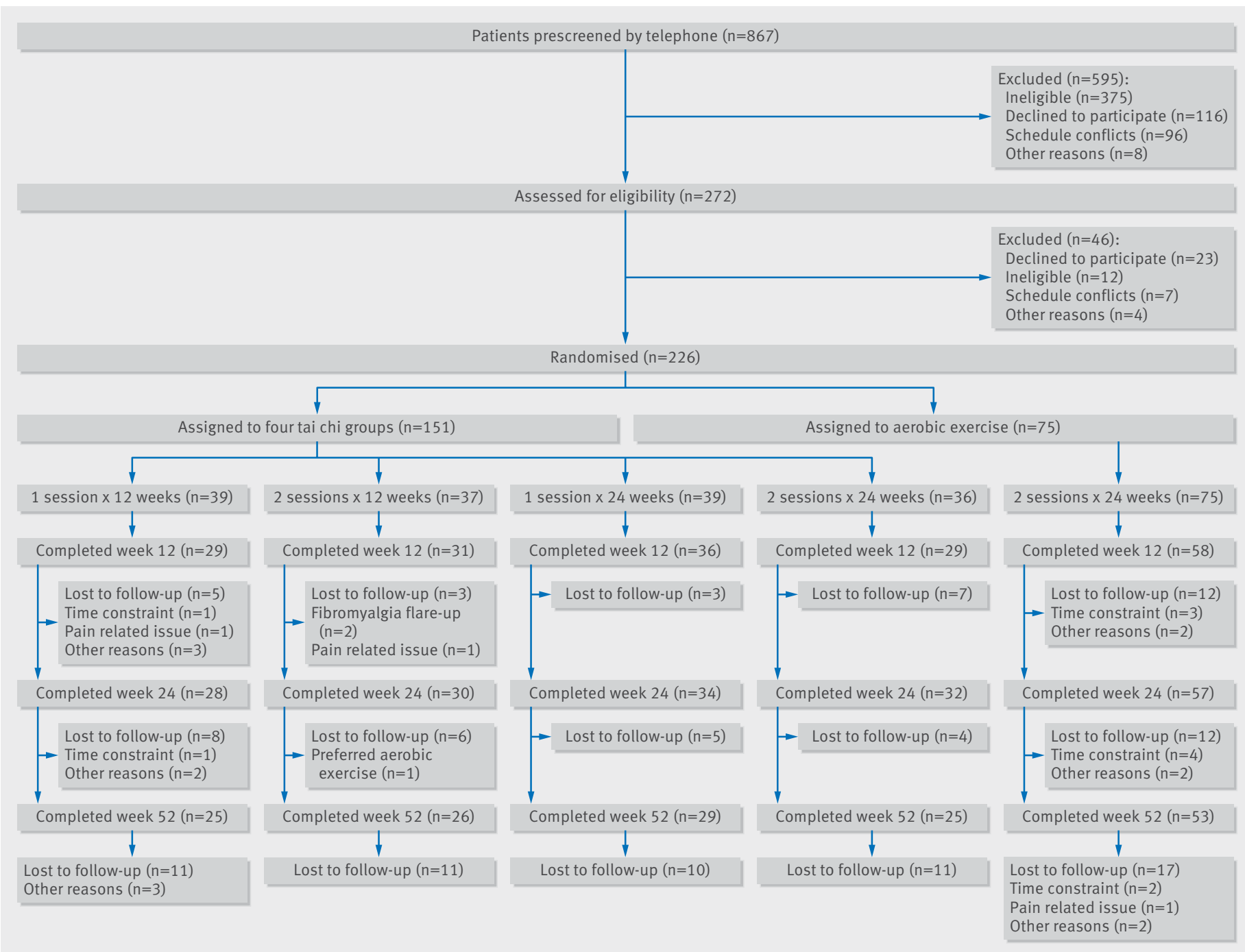

Fig 1 | Eligibility, randomization, and follow-up of study participants

the minimal clinically important difference compared with baseline.

Several secondary outcomes (patient's global assessment 0.9 points, 95\% confidence interval 0.3 to $1.4, \mathrm{P}=0.005$; HADS anxiety 1.2 points, 0.3 to 2.1 , $\mathrm{P}=0.006$; self efficacy 1.0 point, 0.5 to $1.6, \mathrm{P}=0.0004$; and coping strategies 2.6 points, 0.8 to $4.3, \mathrm{P}=0.005$ ) also differed significantly between the combined tai chi and aerobic exercise groups at 24 weeks.

FIQR scores at 24 weeks improved significantly more in the 24 week tai chi groups than 12 week groups (9.6 points, 2.6 to $16.6, \mathrm{P}=0.007$ ). This improvement decreased at 52 weeks. The 24 week tai chi groups also had a significantly larger improvement in scores on HADS depression (1.4 points, 0.1 to $2.6, \mathrm{P}=0.04$ ), Beck depression inventory II (4.4 points, 0.9 to 7.9 , $\mathrm{P}=0.01$ ), and SF-36 mental component (4.4 points, 0.8 to $8.1, \mathrm{P}=0.02$ ) compared with the 12 week groups. In general, treatment effects were less at 52 weeks than at 24 weeks. No significant differences were found at 24 weeks between the groups receiving tai chi once weekly versus twice weekly.
At 52 weeks the combined tai chi groups continued to show more improvement in most primary and secondary outcomes than the aerobic exercise group. Results were statistically significant for patient's global assessment, HADS anxiety, self efficacy, and coping strategies. For the most intensive dose of tai chi (24 weeks, twice weekly), the FIQR score was significantly better for tai chi than for aerobic exercise (11.1 points, 2.7 to $19.6, \mathrm{P}=0.01$ ). The significant benefits were also found for patient's global assessment, HADS anxiety, self efficacy, and coping strategies. In general, all other secondary outcomes favored tai chi compared with aerobic exercise, although the effects were not statistically significant.

The beneficial effects of tai chi were consistent across tai chi instructors. Participants reported 154 adverse events: 117 among 151 participants assigned to tai chi and 37 among 75 participants assigned to aerobic exercise (see appendix table 5). Twelve of these were related to the interventions (eight tai chi, four aerobic exercise), but most were minor musculoskeletal events. Ten serious adverse events were reported 
Table 1 | Baseline characteristics of trial participants, by treatment group. Values are numbers (percentages) unless stated otherwise

\begin{tabular}{|c|c|c|c|c|c|}
\hline \multirow[b]{2}{*}{ Variables } & \multicolumn{4}{|c|}{ Tai chi $(n=151)$} & \multirow{2}{*}{$\begin{array}{l}\text { Aerobic exercise } \\
2 \times 24 \text { weeks } \\
(n=75)\end{array}$} \\
\hline & $\begin{array}{l}1 \times 12 \text { weeks } \\
(n=39)\end{array}$ & $\begin{array}{l}2 \times 12 \text { weeks } \\
(n=37)\end{array}$ & $\begin{array}{l}1 \times 24 \text { weeks } \\
(n=39)\end{array}$ & $\begin{array}{l}2 \times 24 \text { weeks } \\
(n=36)\end{array}$ & \\
\hline Mean (SD) age (years) & $53.0(12.6)$ & $52.1(10.3)$ & $50.8(11.8)$ & $52.1(13.3)$ & $50.9(12.5)$ \\
\hline Women & $33(84.6)$ & $30(81.1)$ & $38(97.4)$ & $36(100.0)$ & $72(96.0)$ \\
\hline White race & $28(71.8)$ & $20(54.1)$ & $24(61.5)$ & $21(58.3)$ & $45(60.0)$ \\
\hline High school or higher education & $38(97.4)$ & $35(94.6)$ & $35(92.1)$ & $35(97.2)$ & $72(96.0)$ \\
\hline Mean (SD) duration of body pain (years) & $11.1(8.8)$ & $12.6(12.1)$ & $12.0(8.3)$ & $13.8(10.4)$ & $11.3(8.7)$ \\
\hline Mean (SD) body mass index $\left(\mathrm{kg} / \mathrm{m}^{2}\right)$ & $30.6(6.4)$ & $30.4(6.8)$ & $29.9(6.4)$ & $29.3(7.4)$ & $30.0(6.8)$ \\
\hline \multicolumn{6}{|l|}{ Drugs: } \\
\hline Paracetamol & $29(74.4)$ & $24(64.9)$ & $25(65.8)$ & $24(66.7)$ & $45(60.0)$ \\
\hline Non-steroidal anti-inflammatory & $34(87.2)$ & $27(73.0)$ & $36(94.7)$ & $31(86.1)$ & $64(85.3)$ \\
\hline Narcotics & $20(51.3)$ & $17(47.2)$ & $22(57.9)$ & $19(52.8)$ & $37(49.3)$ \\
\hline Antidepressant & $22(56.4)$ & $22(59.5)$ & $26(70.3)$ & $14(38.9)$ & $48(64.0)$ \\
\hline Anticonvulsants & $13(33.3)$ & $11(29.7)$ & $7(18.4)$ & $8(22.2)$ & $29(38.7)$ \\
\hline Muscle relaxants & $7(18.0)$ & $11(29.7)$ & $12(31.6)$ & $9(25.0)$ & $22(29.3)$ \\
\hline Benzodiazepines & $14(35.9)$ & $8(21.6)$ & $13(34.2)$ & $7(19.4)$ & $29(38.7)$ \\
\hline \multicolumn{6}{|l|}{ Self reported comorbidities: } \\
\hline Heart disease & $7(18.0)$ & $4(10.8)$ & $1(2.6)$ & $5(13.9)$ & $4(5.3)$ \\
\hline Hypertension & $8(20.5)$ & $17(46.0)$ & $14(36.8)$ & $16(44.4)$ & $13(17.3)$ \\
\hline Diabetes & $5(12.8)$ & $5(13.5)$ & $2(5.3)$ & $5(13.9)$ & $1(1.3)$ \\
\hline Mean (SD) FIQR score (range 0-100)* & $52.4(18.7)$ & $53.8(23.3)$ & $56.5(15.5)$ & $60.4(17.8)$ & $57.3(20.3)$ \\
\hline Mean (SD) symptom severity scale score (range $0-12)^{\star}$ & $8.1(1.9)$ & $8.7(2.1)$ & $8.6(2.2)$ & $9.0(1.7)$ & $8.7(2.0)$ \\
\hline Mean (SD) patient's global assessment score (range $0-10)^{\star}$ & $5.6(2.0)$ & $6.1(1.9)$ & $6.3(1.8)$ & $5.9(1.9)$ & $6.2(2.0)$ \\
\hline \multicolumn{6}{|l|}{ Mean (SD) HADS scores (subscale range 0-21)*: } \\
\hline Depression & $6.9(3.6)$ & $7.4(4.3)$ & $7.6(3.9)$ & $8.5(4.2)$ & $7.6(4.4)$ \\
\hline Anxiety & $9.8(4.8)$ & $8.4(3.7)$ & $8.5(4.4)$ & $9.5(4.6)$ & $8.8(3.8)$ \\
\hline Mean (SD) Beck depression inventory II score (range 0-63) ${ }^{\star}$ & $21.1(12.0)$ & $18.9(11.1)$ & $22.0(12.3)$ & $24.1(14.1)$ & $23.1(11.9)$ \\
\hline Mean (SD) coping strategies questionnaire score (range $0-36) \dagger$ & $14.3(9.9)$ & $15.3(8.6)$ & $14.9(8.6)$ & $17.8(8.9)$ & $15.5(9.0)$ \\
\hline Mean (SD) MOS social support survey score (range 0-5)† & $3.3(1.0)$ & $3.3(1.0)$ & $3.3(1.1)$ & $3.2(1.2)$ & $3.2(1.0)$ \\
\hline Mean (SD) arthritis self efficacy scale score (range 1-10)† & $5.0(1.8)$ & $4.9(2.4)$ & $4.9(2.2)$ & $5.1(2.1)$ & $5.4(2.1)$ \\
\hline Mean (SD) Pittsburgh sleep quality index score (range 0-21)* & $11.6(4.0)$ & $11.6(5.1)$ & $12.0(4.1)$ & $11.3(3.7)$ & $12.5(4.0)$ \\
\hline \multicolumn{6}{|l|}{ Mean (SD) SF-36 scores (range 0-100)t: } \\
\hline Mental component & $39.5(10.5)$ & $42.0(11.1)$ & $40.9(11.9)$ & $39.1(9.8)$ & $39.4(11.1)$ \\
\hline Physical component & $32.8(6.6)$ & $32.5(9.7)$ & $28.5(7.3)$ & $28.5(6.5)$ & $30.3(7.5)$ \\
\hline Mean (SD) 6 minute walk test score $(\mathrm{m})$ & $399.5(80.7)$ & $400.7(71.0)$ & $394.4(81.1)$ & $387.7(87.2)$ & $406.1(90.9)$ \\
\hline Mean (SD) improved HAQ score (range $0-100)^{\star}$ & $18.3(13.2)$ & $26.5(20.1)$ & $23.8(16.5)$ & $26.7(17.9)$ & $26.0(19.9)$ \\
\hline Mean (SD) outcome expectations scale score (range 1-5)† & $3.8(0.7)$ & $3.8(0.9)$ & $3.7(0.8)$ & $3.7(0.9)$ & $3.8(0.8)$ \\
\hline
\end{tabular}

(seven tai chi and three aerobic exercise), but none of these were related to the interventions. These included three cases of cancer, two cases of pneumonia, one case of aseptic meningitis, one case of chicken pox, two cases of emergency hysterectomy, and one case of concussion. No patients withdrew from the study because of adverse events.

All tai chi and aerobic exercise groups showed similar reduced use of analgesics, antidepressants, muscle relaxants, and antiepileptic drugs over time (see appendix table 6).

Several sensitivity analyses examined the effect of missing observations. Comparisons of baseline characteristics between participants with missing versus complete data at each evaluation visit revealed differences in age, duration of body pain, self efficacy, FIQR score, Pittsburgh sleep quality index, SF-36 mental component, HADS anxiety, six minute walk test, and attendance rate (see appendix table 2) at one or more times. Adjustment for these variables and attendance rate in longitudinal analyses and additional sensitivity analyses did not substantively change the primary results. Use of multiple imputation led to small changes in some of the treatment effects (see appendix table 3). Although these changes were not consistently in any one direction, they did attenuate the effects of a few of the treatment comparisons, including the primary outcome (see appendix table 4), the $P$ value for which increased to 0.08 . The subgroup analysis of participants who attended at least $50 \%$ sessions in both the aerobic exercise and the tai chi groups showed similar effects as in the entire group, although statistical significance was reduced because of the smaller subgroup sample size (see appendix table 4). Regression model diagnostics supported model assumptions of normality and homoscedasticity and did not show evidence of any influential data points.

\section{Discussion}

This trial found that tai chi mind-body intervention results in similar or greater symptom improvement compared with aerobic exercise, the current most commonly prescribed non-drug treatment for patients with fibromyalgia. Participants in the combined tai 
Table 2 | Mean change ( $95 \%$ confidence interval) from baseline in primary and secondary outcomes

Tai chi ( $n=151)$

Variables

$1 \times 12$ weeks $(n=39)$

$2 \times 12$ weeks $(n=37)$

$1 \times 24$ weeks $(n=39)$

$2 \times 24$ weeks $(n=36)$

Aerobic exercise $2 \times 24$ weeks $(n=75)$

FIQR score (range 0-100)*:

\begin{tabular}{|c|c|c|c|c|c|}
\hline Week 12 & $-16.5(-23.4$ to -9.6$)$ & $-12.3(-19.0$ to -5.6$)$ & $-6.6(-12.8$ to -0.3$)$ & $-17.2(-24.0$ to -10.3$)$ & $-6.2(-11.0$ to -1.4$)$ \\
\hline Week 24 & $-11.4(-18.7$ to -4.1$)$ & $-11.4(-18.4$ to -4.4$)$ & $-16.7(-23.4$ to -10.1$)$ & $-25.4(-32.3$ to -18.4$)$ & $-9.2(-14.3$ to -4.1$)$ \\
\hline Week 52 & $-14.3(-21.7$ to -7.0$)$ & $-10.2(-17.3$ to -3.1$)$ & $-13.6(-20.4$ to -6.8$)$ & $-22.7(-30.0$ to -15.4$)$ & $-11.7(-16.7$ to -6.6$)$ \\
\hline \multicolumn{6}{|c|}{$\begin{array}{l}\text { Symptom severity scale score } \\
\text { (range 0-12)*: }\end{array}$} \\
\hline Week 12 & $-1.0(-1.8$ to -0.2$)$ & $-1.7(-2.5$ to -0.9$)$ & $-1.1(-1.8$ to -0.3$)$ & $-1.4(-2.2$ to -0.6$)$ & $-0.7(-1.3$ to -0.1$)$ \\
\hline Week 24 & $-0.5(-1.4$ to 0.4$)$ & $-2.0(-2.9$ to -1.2$)$ & $-1.8(-2.6$ to -1.0$)$ & $-1.7(-2.5$ to -0.8$)$ & $-0.8(-1.4$ to -0.2$)$ \\
\hline Week 52 & $-1.0(-1.9$ to 0.0$)$ & $-2.5(-3.5$ to -1.6$)$ & $-1.4(-2.3$ to -0.6$)$ & $-1.8(-2.8$ to -0.9$)$ & $-1.1(-1.8$ to -0.4$)$ \\
\hline
\end{tabular}

Patient's global assessment score

(range 0-10)*:

\begin{tabular}{|c|c|c|c|c|c|}
\hline Week 12 & $-1.5(-2.4$ to -0.7$)$ & $-1.4(-2.2$ to -0.6$)$ & $-1.1(-1.8$ to -0.3$)$ & $-0.6(-1.5$ to 0.2$)$ & $-0.4(-1.0$ to 0.2$)$ \\
\hline Week 24 & $-1.0(-1.8$ to -0.1$)$ & $-1.3(-2.2$ to -0.5$)$ & $-1.6(-2.4$ to -0.8$)$ & $-2.0(-2.8$ to -1.2$)$ & $-0.4(-1.0$ to 0.2$)$ \\
\hline Week 52 & $-0.7(-1.6$ to 0.3$)$ & $-1.2(-2.2$ to -0.3$)$ & $-1.4(-2.2$ to -0.5$)$ & $-1.7(-2.7$ to -0.8$)$ & $-0.3(-0.9$ to 0.3$)$ \\
\hline \multicolumn{6}{|c|}{ HADS scores (subscale range $0-21)^{\star}$ : } \\
\hline \multicolumn{6}{|c|}{ Depression: } \\
\hline Week 12 & $-1.2(-2.3$ to- -0.1$)$ & $-1.3(-2.3$ to- -0.2$)$ & $-0.9(-1.9$ to 0.1$)$ & $-1.7(-2.8$ to 0.6$)$ & $-0.5(-1.3$ to 0.3$)$ \\
\hline Week 24 & $-0.7(-2.0$ to 0.6$)$ & $-0.7(-2.0$ to 0.5$)$ & $-1.4(-2.6$ to 0.3$)$ & $-2.7(-4.1$ to 1.4$)$ & $-0.6(-1.5$ to 0.4$)$ \\
\hline Week 52 & $-2.0(-3.4$ to 0.5$)$ & $-1.7(-3.1$ to 0.3$)$ & $-0.9(-2.2$ to 0.5$)$ & $-2.2(-3.7$ to 0.8$)$ & $-0.6(-1.6$ to 0.4$)$ \\
\hline \multicolumn{6}{|l|}{ Anxiety: } \\
\hline Week 12 & $-1.7(-2.8$ to -0.6$)$ & $-0.8(-1.9$ to 0.2$)$ & $-1.1(-2.1$ to -0.1$)$ & $-1.6(-2.7$ to -0.4$)$ & $0.2(-0.6$ to 1.0$)$ \\
\hline Week 24 & $-1.9(-3.2$ to -0.7$)$ & $-0.8(-2.0$ to 0.4$)$ & $-1.4(-2.5$ to -0.2$)$ & $-2.1(-3.4$ to -0.8$)$ & $0.0(-0.9$ to 0.9$)$ \\
\hline Week 52 & $-2.4(-3.9$ to -1.0$)$ & $-2.2(-3.6$ to -0.8$)$ & $-1.3(-2.7$ to 0.0$)$ & $-2.1(-3.6$ to -0.7$)$ & $-0.4(-1.4$ to 0.6$)$ \\
\hline
\end{tabular}

Beck depression inventory II score

(range 0-63)*:

\begin{tabular}{clllll}
\hline Week 12 & $-6.0(-9.7$ to -2.2$)$ & $-5.8(-9.4$ to -2.1$)$ & $-3.7(-7.1$ to -0.3$)$ & $-7.4(-11.1$ to -3.6$)$ & $-3.9(-6.5$ to -1.3$)$ \\
\hline Week 24 & $-3.8(-7.5$ to -0.2$)$ & $-4.3(-7.8$ to -0.8$)$ & $-7.5(-10.8$ to -4.1$)$ & $-9.5(-13.0$ to -6.0$)$ & $-5.2(-7.7$ to -2.7$)$ \\
\hline Week 52 & $-7.6(-11.8$ to -3.4$)$ & $-7.5(-11.7$ to -3.3$)$ & $-5.5(-9.4$ to -1.6$)$ & $-11.1(-15.2$ to -6.9$)$ & $-6.4(-9.3$ to -3.5$)$ \\
\hline Coping strategies questionnaire & & & &
\end{tabular}

Coping strategies questionnaire

score (range 0-36)t:

\begin{tabular}{|c|c|c|c|c|c|}
\hline Week 12 & $-4.0(-6.4$ to -1.5$)$ & $-4.0(-6.4$ to -1.6$)$ & $-3.2(-5.5$ to -1.0$)$ & $-6.3(-8.8$ to -3.8$)$ & $-1.5(-3.2$ to 0.3$)$ \\
\hline Week 24 & $-4.7(-7.2$ to -2.2$)$ & $-4.1(-6.5$ to -1.6$)$ & $-3.9(-6.3$ to -1.6$)$ & $-6.8(-9.5$ to -4.2$)$ & $-1.6(-3.4$ to 0.2$)$ \\
\hline Week 52 & $-5.4(-8.1$ to -2.6$)$ & $-5.0(-7.7$ to -2.4$)$ & $-5.1(-7.7$ to -2.4$)$ & $-7.2(-10.0$ to -4.4$)$ & $-2.1(-4.0$ to -0.2$)$ \\
\hline \multicolumn{6}{|c|}{$\begin{array}{l}\text { MOS social support survey score } \\
\text { (range 0-5)t: }\end{array}$} \\
\hline Week 12 & $0.1(-0.2$ to 0.3$)$ & $0.3(0.1$ to 0.6$)$ & $0.0(-0.2$ to 0.3$)$ & $-0.1(-0.3$ to 0.2$)$ & $0.0(-0.1$ to 0.2$)$ \\
\hline Week 24 & $0.0(-0.3$ to 0.3$)$ & $0.3(0.0$ to 0.6$)$ & $0.1(-0.2$ to 0.4$)$ & $0.1(-0.2$ to 0.4$)$ & $0.1(-0.1$ to 0.3$)$ \\
\hline Week 52 & $0.1(-0.3$ to 0.4$)$ & $0.5(0.1$ to 0.8$)$ & $0.3(0.0$ to 0.6$)$ & $0.0(-0.3$ to 0.4$)$ & $0.2(0.0$ to 0.5$)$ \\
\hline \multicolumn{6}{|c|}{$\begin{array}{l}\text { Arthritis self efficacy scale score } \\
\text { (range 1-10)t: }\end{array}$} \\
\hline Week 12 & $1.0(0.1$ to 1.8$)$ & $1.3(0.5$ to 2.1$)$ & $0.3(-0.5$ to 1.1$)$ & $1.1(0.2$ to 1.9$)$ & $-0.1(-0.7$ to 0.5$)$ \\
\hline Week 24 & $0.8(0.0$ to 1.7$)$ & $1.1(0.3$ to 1.9$)$ & $1.5(0.7$ to 2.2$)$ & $1.5(0.6$ to 2.3$)$ & $-0.1(-0.7$ to 0.5$)$ \\
\hline Week 52 & $0.8(-0.1$ to 1.6$)$ & 1.9 (1.1 to 2.8$)$ & $1.2(0.3$ to 2.0$)$ & $1.1(0.2$ to 2.0$)$ & $-0.1(-0.7$ to 0.5$)$ \\
\hline \multicolumn{6}{|c|}{$\begin{array}{l}\text { Pittsburgh sleep quality index score } \\
\text { (range, } 0-21)^{\star} \text { : }\end{array}$} \\
\hline Week 12 & $-1.0(-2.2$ to 0.2$)$ & $-1.1(-2.3$ to 0.1$)$ & $-0.6(-1.7$ to 0.5$)$ & $-1.6(-2.8$ to -0.4$)$ & $-0.9(-1.7$ to -0.1$)$ \\
\hline Week 24 & $-0.8(-2.2$ to 0.6$)$ & $-1.3(-2.7$ to 0.1$)$ & $-1.9(-3.2$ to -0.6$)$ & $-2.1(-3.5$ to -0.7$)$ & $-1.1(-2.1$ to -0.1$)$ \\
\hline Week 52 & $-2.6(-4.2$ to -1.1$)$ & $-2.2(-3.8$ to -0.7$)$ & $-1.1(-2.6$ to 0.4$)$ & $-2.0(-3.6$ to -0.4$)$ & $-1.2(-2.3$ to -0.1$)$ \\
\hline \multicolumn{6}{|c|}{ SF-36 scores (range 0-100)t: } \\
\hline \multicolumn{6}{|c|}{ Mental component: } \\
\hline Week 12 & 4.5 (0.7 to 8.2$)$ & $3.2(-0.4$ to 6.8$)$ & $3.2(-0.3$ to 6.6$)$ & 3.8 (0.0 to 7.6$)$ & $0.6(-2.1$ to 3.3$)$ \\
\hline Week 24 & $3.2(-0.4$ to 6.9$)$ & $0.3(-3.2$ to 3.9$)$ & $5.3(1.9$ to 8.7$)$ & 7.4 (3.6 to 11.2$)$ & $0.9(-1.8$ to 3.6$)$ \\
\hline Week 52 & $5.4(0.9$ to 9.9$)$ & 5.7 (1.4 to 10.0$)$ & $3.8(-0.5$ to 8.0$)$ & $5.4(0.8$ to 9.9$)$ & $3.0(-0.1$ to 6.0$)$ \\
\hline \multicolumn{6}{|c|}{ Physical component: } \\
\hline Week 12 & $3.9(1.3$ to 6.4$)$ & 3.8 (1.4 to 6.2$)$ & $1.9(-0.4$ to 4.2$)$ & $3.3(0.7$ to 5.8$)$ & $1.8(-0.1$ to 3.6$)$ \\
\hline Week 24 & $2.4(-0.4$ to 5.2$)$ & $3.9(1.2$ to 6.6$)$ & $5.0(2.5$ to 7.6$)$ & 5.9 (3.1 to 8.8) & $4.0(2.0$ to 6.0$)$ \\
\hline Week 52 & $4.5(1.4$ to 7.7$)$ & 3.7 (0.6 to 6.7) & 6.9 (3.9 to 9.9) & $5.4(2.2$ to 8.6$)$ & $2.6(0.4$ to 4.7$)$ \\
\hline \multicolumn{6}{|c|}{6 minute walk test score $(\mathrm{m})$ : } \\
\hline Week 12 & $16.1(-5.3$ to 37.5$)$ & $29.7(9.2$ to 50.3$)$ & $16.0(-3.7$ to 35.7$)$ & $7.4(-14.8$ to 29.6$)$ & $9.3(-6.1$ to 24.8$)$ \\
\hline Week 24 & $29.0(7.4$ to 50.6$)$ & $31.6(9.8$ to 53.3$)$ & $23.2(2.6$ to 43.7$)$ & $17.7(-5.1$ to 40.4$)$ & $18.0(1.8$ to 34.2$)$ \\
\hline Week 52 & $23.8(-7.0$ to 54.6$)$ & $7.0(-22.4$ to 36.3$)$ & $34.9(5.6$ to 64.2$)$ & $30.2(-1.6$ to 61.9$)$ & $8.0(-13.3$ to 29.4$)$ \\
\hline \multicolumn{6}{|c|}{ Body mass index: } \\
\hline Week 12 & $0.1(-0.3$ to 0.6$)$ & $-0.1(-0.5$ to 0.4$)$ & $-0.2(-0.6$ to 0.2$)$ & $0.1(-0.3$ to 0.6$)$ & $-0.1(-0.4$ to 0.2$)$ \\
\hline Week 24 & $0.3(-0.3$ to 0.8$)$ & $-0.1(-0.6$ to 0.4$)$ & $0.1(-0.4$ to 0.6$)$ & $0.3(-0.3$ to 0.8$)$ & $0.1(-0.3$ to 0.5$)$ \\
\hline Week 52 & $0.5(-0.4 .1 .4)$ & $-0.2(-1.1$ to 0.6$)$ & $0.1(-0.8$ to 0.9$)$ & $0.6(-0.3$ to 1.5$)$ & $-0.2(-0.8$ to 0.4$)$ \\
\hline
\end{tabular}


Table 2 | Mean change (95\% confidence interval) from baseline in primary and secondary outcomes (Continued)

\begin{tabular}{|c|c|c|c|c|c|}
\hline \multirow[b]{2}{*}{ Variables } & \multicolumn{4}{|l|}{ Tai chi $(n=151)$} & \multirow{2}{*}{$\begin{array}{l}\text { Aerobic exercise } 2 \times 24 \\
\text { weeks }(n=75)\end{array}$} \\
\hline & $1 \times 12$ weeks $(n=39)$ & $2 \times 12$ weeks $(n=37)$ & $1 \times 24$ weeks $(n=39)$ & $2 \times 24$ weeks $(n=36)$ & \\
\hline \multicolumn{6}{|c|}{ Improved HAQ score (range 0-100)*: } \\
\hline Week 12 & $-2.0(-6.5$ to 2.6$)$ & $-9.2(-13.6$ to -4.8$)$ & $-0.4(-4.6$ to 3.8$)$ & $-6.4(-11.0$ to -1.8$)$ & $-1.9(-5.1$ to 1.3$)$ \\
\hline Week 24 & $-2.5(-7.6$ to 2.6$)$ & $-3.1(-8.3$ to 2.1$)$ & $-3.9(-8.6$ to 0.9$)$ & $-6.7(-12.0$ to -1.3$)$ & $-4.1(-7.8$ to -0.5$)$ \\
\hline Week 52 & $-3.3(-8.9$ to 2.3$)$ & $-9.5(-14.9$ to -4.1$)$ & $-3.5(-8.8$ to 1.8$)$ & $-5.0(-10.8$ to 0.7$)$ & $-3.9(-7.8$ to 0.0$)$ \\
\hline \multicolumn{6}{|c|}{$\begin{array}{l}\text { Outcome expectations scale score } \\
\text { (range 1-5)t: }\end{array}$} \\
\hline Week 12 & $0.2(-0.2$ to 0.5$)$ & $0.1(-0.3$ to 0.4$)$ & $0.2(-0.1$ to 0.5$)$ & $0.4(0.0$ to 0.7$)$ & $0.1(-0.2$ to 0.3$)$ \\
\hline Week 24 & $0.0(-0.3$ to 0.3$)$ & $0.1(-0.2$ to 0.4$)$ & $0.2(0.0$ to 0.5$)$ & $0.3(0.0$ to 0.6$)$ & $0.1(-0.2$ to 0.3$)$ \\
\hline Week 52 & $0.0(-0.3$ to 0.3$)$ & $0.2(-0.1$ to 0.5$)$ & $0.1(-0.2$ to 0.4$)$ & $0.2(-0.1$ to 0.6$)$ & $0.0(-0.2$ to 0.2$)$ \\
\hline
\end{tabular}

chi groups showed significantly more improvement in the primary outcome (change in FIQR scores between baseline and 24 weeks), although the difference became non-significant on one sensitivity analysis using multiple imputation. With the same intensity and duration (24 weeks, twice weekly), tai chi showed a larger clinically important effect for the primary outcome and significant effects for many secondary outcomes compared with aerobic exercise. Notably, a longer duration of tai chi (24 weeks) had more benefit than a shorter duration (12 weeks). At 24 weeks, participants in the 24 week tai chi groups had significantly more improvement in FIQR score than those in the 12 week groups. These gains were smaller and non-significant at 52 weeks. As expected, the groups did not differ at 12 weeks since the interventions were identical up to that point. Participants assigned to the mind-body therapy also attended training classes more often than those assigned to aerobic exercise. No serious adverse events related to the interventions were reported in the participants.

\section{Comparison with other studies}

The results of this trial are consistent with those of our previous attention controlled study and other small, shorter efficacy trials that showed the benefits of mindbody practices such as tai chi, qigong, and yoga for pain and physical and psychological health, compared with various interventions for fibromyalgia and other chronic pain conditions. ${ }^{23} 246263$ These findings suggest an important alternative strategy for managing fibromyalgia.

Substantial evidence in the past several decades has suggested aerobic exercise (on land and in water) and its combinations effectively treat fibromyalgia and other chronic musculoskeletal pain disorders. ${ }^{9-19}$ Continuing exercise maintains positive effects on pain, depressed mood, physical fitness, and health related quality of life. However, some patients with fibromyalgia have difficulties performing and adhering to exercise programs, indicating a need for other effective therapeutic options. ${ }^{15}$ In this study, participants assigned to the mind-body therapy maintained higher and more consistent attendance than those assigned to aerobic exercise. Tai chi, which consists of a gentler, low impact meditative sequence of movements with minimal side effects, may be better embraced by patients with fibromyalgia in the long term.

Fibromyalgia has been primarily recognized as a complex central pain disorder influencing pain perception and is characterized by a low threshold of pain modulated by emotion, thoughts, and environment. Tai chi, a multicomponent intervention that integrates physical, psychosocial, emotional, spiritual, and behavioral elements, aims at inducing effects on both body and mind that may result in beneficial health outcomes. ${ }^{33}$ More clinical trials are needed to confirm whether behavioral interventions such as mind-body exercise can improve central pain sensitization and symptom management in the fibromyalgia population.

By improving psychological wellbeing, coping, and self efficacy, tai chi mind-body exercise may help to bolster the confidence of patients with fibromyalgia to engage in behaviors that help them manage their symptoms and to persist in those behaviors. ${ }^{64} 65$ Tai chi might also help buffer the negative impact of fibromyalgia symptoms on the patients physical and psychosocial wellbeing. ${ }^{65}$ At the highest intensity and longest duration of interventions (24 weeks, twice weekly), our results revealed significant differences in improvements in overall severity of fibromyalgia symptoms as well as depression, anxiety, self efficacy, and mental component of health related quality of life between tai chi and aerobic exercise. In addition, pain symptoms of fibromyalgia have been closely linked to concurrent mood disorders. Compared with the 12 week tai chi groups, our study revealed that the 24 week tai chi groups achieved significant improvements in depression and mental health, indicating that psychological benefits might be associated with longer mind-body practice. In light of these complex multicomponent mind-body interactions on and with various active behavioral elements, tai chi may be especially well suited to the treatment of fibromyalgia.

\section{Strengths and limitations of this study}

The strengths of this trial include the large and diverse sample of participants with fibromyalgia, including 
Table 3 | Between group differences in primary and secondary outcomes at all weeks*

Aerobic exercise $v$ tai chi groups

\section{combined}

Variables

FIQR score

(range 0-100)†:

Week 12

Week 24

Week 52

Mean $(95 \% \mathrm{Cl})$

$P$ value

Tai chi: 12 v 24 weeks

Mean $(95 \% \mathrm{Cl})$
Tai chi: once $v$ twice weekly

$P$ value

\section{Mean $(95 \% \mathrm{Cl})$}

Aerobic exercise $2 \times 24$ weeks $v$ tai chi $2 \times 24$ weeks Mean $(95 \% \mathrm{Cl})$

$P$ value

Symptom severity scale

score (range 0-12)t:

\begin{tabular}{|c|c|c|c|c|c|c|c|c|}
\hline Week 12 & $0.5(-0.1$ to 1.0$)$ & 0.11 & $-0.2(-0.9$ to 0.6$)$ & 0.70 & $0.5(-0.3$ to 1.3$)$ & 0.20 & $0.6(-0.3$ to 1.5$)$ & 0.17 \\
\hline Week 24 & $0.5(-0.1$ to 1.1$)$ & 0.07 & $0.5(-0.4$ to 1.3$)$ & 0.29 & $0.7(-0.2$ to 1.5$)$ & 0.12 & $0.9(-0.1$ to 1.9$)$ & 0.09 \\
\hline Week 52 & $0.5(-0.2$ to 1.1$)$ & 0.14 & $-0.1(-1.1$ to 0.8$)$ & 0.80 & $1.0(-0.0$ to 1.9$)$ & 0.047 & $0.7(-0.3$ to 1.8$)$ & 0.18 \\
\hline \multicolumn{9}{|c|}{$\begin{array}{l}\text { Patient's global } \\
\text { assessment score } \\
\text { (range } 0-10) \dagger:\end{array}$} \\
\hline Week 12 & 0.6 (0.1 to 1.2$)$ & 0.03 & $-0.6(-1.4$ to 0.2$)$ & 0.12 & $-0.2(-1.0$ to 0.6$)$ & 0.54 & $0.3(-0.6$ to 1.1$)$ & 0.57 \\
\hline Week 24 & 0.9 (0.3 to 1.4$)$ & 0.005 & $0.7(-0.2$ to 1.5$)$ & 0.12 & $0.4(-0.4$ to 1.2$)$ & 0.35 & 1.6 (0.7 to 2.5$)$ & 0.0006 \\
\hline Week 52 & $0.8(0.2$ to 1.4$)$ & 0.01 & $0.6(-0.3$ to 1.5$)$ & 0.17 & $0.5(-0.4$ to 1.4$)$ & 0.30 & $1.5(0.4$ to 2.5$)$ & 0.008 \\
\hline
\end{tabular}

HADS scores

$5.4(0.6$ to 10.1$)$

5.5 (0.6 to 10.4$)$

2.7 ( -2.3 to 7.7$)$

$0.44 \quad 3.4(-3.3$ to 10.1$)$

$\mathbf{0 . 0 0 7} \quad 4.5(-2.5$ to 11.4$)$

$0.11 \quad 2.7$ ( -4.5 to 9.9 )
0.32

0.21

0.46

$10.9(3.4$ to 18.5$)$

0.005

$<0.001$

0.01

(subscale range 0-21)t:

Depression:

\begin{tabular}{|c|c|c|c|c|c|c|c|c|}
\hline Week 12 & $0.6(-0.2$ to 1.4$)$ & 0.12 & $0.1(-1.0$ to 1.2$)$ & 0.90 & $0.5(-0.6$ to 1.5$)$ & 0.41 & $1.1(-0.1$ to 2.4$)$ & 0.07 \\
\hline Week 24 & $0.7(-0.3$ to 1.6$)$ & 0.16 & $1.4(0.1$ to 2.6$)$ & 0.04 & 0.7 (-0.6 to 1.9$)$ & 0.31 & 2.1 (0.5 to 3.7) & 0.01 \\
\hline Week 52 & $0.8(-0.1$ to 1.8$)$ & 0.09 & $-0.3(-1.7$ to 1.2$)$ & 0.71 & $0.5(-0.9$ to 2.0$)$ & 0.46 & 1.6 (0.0 to 3.2$)$ & 0.05 \\
\hline \multicolumn{9}{|l|}{ Anxiety: } \\
\hline Week 12 & 1.2 (0.4 to 2.0$)$ & 0.003 & $0.0(-1.0$ to 1.1$)$ & 0.93 & $-0.2(-1.3$ to 0.9$)$ & 0.76 & 1.7 (0.3 to 3.1) & 0.02 \\
\hline Week 24 & $1.2(0.3$ to 2.1$)$ & 0.006 & $0.4(-0.8$ to 1.6$)$ & 0.55 & $-0.2(-1.4$ to 1.0$)$ & 0.74 & 2.1 (0.6 to 3.6$)$ & 0.008 \\
\hline Week 52 & 1.3 (0.3 to 2.2$)$ & 0.009 & $-0.6(-2.0$ to 0.8$)$ & 0.41 & $0.3(-1.1$ to 1.7$)$ & 0.67 & $1.6(0.1$ to 3.1$)$ & 0.04 \\
\hline \multicolumn{9}{|c|}{$\begin{array}{l}\text { Beck depression } \\
\text { inventory II score } \\
\text { (range 0-63)†: }\end{array}$} \\
\hline Week 12 & $1.4(-1.1$ to 3.9$)$ & 0.28 & $-0.4(-4.0$ to 3.2$)$ & 0.84 & $1.8(-1.8$ to 5.4$)$ & 0.34 & $3.3(-0.9$ to 7.5$)$ & 0.12 \\
\hline Week 24 & $0.9(-1.6$ to 3.3$)$ & 0.49 & $4.4(0.9$ to 7.9$)$ & 0.01 & $1.2(-2.3$ to 4.7$)$ & 0.49 & $4.3(0.0$ to 8.5$)$ & 0.049 \\
\hline Week 52 & $1.2(-1.6$ to 4.1$)$ & 0.40 & $0.6(-3.5$ to 4.8$)$ & 0.76 & $2.8(-1.3$ to 6.9$)$ & 0.18 & $4.6(-0.5$ to 9.7$)$ & 0.08 \\
\hline \multicolumn{9}{|c|}{$\begin{array}{l}\text { Coping strategies score } \\
\text { (range 0-36)‡: }\end{array}$} \\
\hline Week 12 & 2.3 (0.6 to 4.0$)$ & 0.009 & $0.8(-1.7$ to 3.2$)$ & 0.54 & $1.6(-0.8$ to 4.0$)$ & 0.20 & 4.8 (1.7 to 8.0$)$ & 0.003 \\
\hline Week 24 & $2.6(0.8$ to 4.3$)$ & 0.005 & $0.9(-1.6$ to 3.4$)$ & 0.46 & $1.1(-1.4$ to 3.6$)$ & 0.38 & $5.4(2.1$ to 8.9$)$ & 0.002 \\
\hline Week 52 & $2.8(1.0$ to 4.7$)$ & 0.003 & $0.9(-1.8$ to 3.6$)$ & 0.50 & $0.9(-1.8$ to 3.6$)$ & 0.50 & $4.9(1.4$ to 8.3$)$ & 0.006 \\
\hline \multicolumn{9}{|c|}{$\begin{array}{l}\text { MOS social support } \\
\text { survey score (range } \\
\text { 0-5)‡: }\end{array}$} \\
\hline Week 12 & $0.0(-0.1$. to 0.2$)$ & 0.65 & $-0.2(-0.5$ to 0.0$)$ & 0.09 & $0.1(-0.2$ to 0.3$)$ & 0.56 & $-0.1(-0.4$ to 0.2$)$ & 0.41 \\
\hline Week 24 & $0.0(-0.2$ to 0.2$)$ & 0.99 & $-0.1(-0.3$ to 0.2$)$ & 0.73 & $0.1(-0.2$ to 0.4$)$ & 0.40 & $0.0(-0.4$ to 0.3$)$ & 0.81 \\
\hline Week 52 & $0.0(-0.2$ to 0.2$)$ & 0.96 & $-0.1(-0.4$ to 0.3$)$ & 0.63 & $0.1(-0.3$ to 0.4$)$ & 0.77 & $-0.2(-0.6$ to 0.1$)$ & 0.24 \\
\hline
\end{tabular}

Arthritis self efficacy

scale score (range

1-10)‡:

\begin{tabular}{|c|c|c|c|c|c|c|c|c|}
\hline Week 12 & $0.8(0.2$ to 1.4$)$ & 0.009 & $-0.5(-1.3$ to 0.3$)$ & 0.24 & $0.6(-0.2$ to 1.4$)$ & 0.17 & $1.2(0.2$ to 2.1$)$ & 0.02 \\
\hline Week 24 & $1.0(0.5$ to 1.6$)$ & 0.0004 & $0.5(-0.3$ to 1.3$)$ & 0.23 & $0.1(-0.7$ to 0.9$)$ & 0.73 & 1.5 (0.6 to 2.5$)$ & 0.002 \\
\hline Week 52 & 1.1 (0.5 to 1.7$)$ & 0.0004 & $-0.2(-1.1$ to 0.7$)$ & 0.66 & $0.6(-0.3$ to 1.4$)$ & 0.21 & $1.2(0.2$ to 2.2$)$ & 0.02 \\
\hline \multicolumn{9}{|c|}{$\begin{array}{l}\text { Pittsburgh sleep quality } \\
\text { index score (range } \\
0-21)+ \text { : }\end{array}$} \\
\hline Week 12 & $0.1(-0.7$ to 0.9$)$ & 0.78 & $0.0(-1.1$ to 1.2$)$ & 0.99 & $0.6(-0.6$ to 1.7$)$ & 0.34 & $0.6(-0.8$ to 2.1$)$ & 0.37 \\
\hline Week 24 & $0.3(-0.6$ to 1.3$)$ & 0.49 & $1.0(-0.4$ to 2.3$)$ & 0.16 & $0.3(-1.0$ to 1.7$)$ & 0.62 & $1.0(-0.6$ to 2.5$)$ & 0.22 \\
\hline Week 52 & $0.6(-0.4$ to 1.7$)$ & 0.24 & $-0.9(-2.5$ to 0.6$)$ & 0.24 & $0.3(-1.2$ to 1.9$)$ & 0.70 & $0.9(-0.7$ to 2.5$)$ & 0.26 \\
\hline \multicolumn{9}{|c|}{$\begin{array}{l}\text { SF-36 scores (range } \\
0-100) \neq:\end{array}$} \\
\hline \multicolumn{9}{|c|}{ Mental component: } \\
\hline Week 12 & $2.4(-0.2$ to 5.0$)$ & 0.07 & $-0.4(-4.0$ to 3.3$)$ & 0.84 & $-0.3(-4.0$ to 3.3$)$ & 0.86 & $3.2(-0.6$ to 6.9$)$ & 0.10 \\
\hline Week 24 & $2.5(-0.1$ to 5.0$)$ & 0.06 & $4.4(0.8$ to 8.1$)$ & 0.02 & $-0.4(-4.0$ to 3.2$)$ & 0.83 & $6.2(1.9$ to 10.6$)$ & 0.006 \\
\hline Week 52 & $1.7(-1.3$ to 4.7$)$ & 0.27 & $-1.0(-5.4$ to 3.4$)$ & 0.66 & $0.9(-3.5$ to 5.3$)$ & 0.68 & $2.2(-2.7$ to 7.1$)$ & 0.38 \\
\hline \multicolumn{9}{|c|}{ Physical component: } \\
\hline Week 12 & $1.1(-0.6$ to 2.9$)$ & 0.21 & $-1.3(-3.7$ to 1.2$)$ & 0.31 & $0.7(-1.8$ to 3.1$)$ & 0.60 & $1.4(-1.4$ to 4.2$)$ & 0.32 \\
\hline Week 24 & $0.3(-1.7$ to 2.2$)$ & 0.79 & $2.4(-0.3$ to 5.1$)$ & 0.09 & $1.2(-1.5$ to 3.9$)$ & 0.38 & $2.0(-1.3$ to 5.3$)$ & 0.24 \\
\hline Week 52 & $2.0(-0.1$ to 4.2$)$ & 0.06 & $2.0(-1.1$ to 5.1$)$ & 0.20 & $-1.2(-4.3$ to 1.9$)$ & 0.45 & $3.0(-0.7$ to 6.8$)$ & 0.11 \\
\hline
\end{tabular}


Table 3 | Between group differences in primary and secondary outcomes at all weeks* (Continued)

\begin{tabular}{|c|c|c|c|c|c|c|c|c|}
\hline \multirow[b]{2}{*}{ Variables } & \multicolumn{2}{|c|}{$\begin{array}{l}\text { Aerobic exercise } v \text { tai chi groups } \\
\text { combined }\end{array}$} & \multicolumn{2}{|l|}{ Tai chi: $12 v 24$ weeks } & \multicolumn{2}{|c|}{ Tai chi: once $v$ twice weekly } & \multicolumn{2}{|c|}{$\begin{array}{l}\text { Aerobic exercise } 2 \times 24 \\
\text { weeks } v \text { tai chi } 2 \times 24 \text { weeks }\end{array}$} \\
\hline & Mean $(95 \% \mathrm{Cl})$ & $P$ value & Mean $(95 \% \mathrm{Cl})$ & P value & Mean $(95 \% \mathrm{Cl})$ & Pvalue & Mean $(95 \% \mathrm{Cl})$ & P value \\
\hline \multicolumn{9}{|c|}{$\begin{array}{l}6 \text { minute walk test } \\
\text { score }(\mathrm{m}) \text { : }\end{array}$} \\
\hline Week 12 & $6.8(-8.1$ to 21.7$)$ & 0.37 & $-10.7(-31.7$ to 10.2$)$ & 0.31 & $2.5(-18.4$ to 23.5$)$ & 0.81 & $-3.0(-26.1$ to 20.2$)$ & 0.45 \\
\hline Week 24 & $6.0(-9.5$ to 21.6$)$ & 0.45 & $-9.3(-30.9$ to 12.2$)$ & 0.39 & $-1.4(-23.0$ to 20.2$)$ & 0.90 & $-1.0(-27.1$ to 25.1$)$ & 0.94 \\
\hline Week 52 & $12.5(-8.4$ to 33.4$)$ & 0.24 & $17.1(-13.2$ to 47.4$)$ & 0.27 & $-10.7(-41.0$ to 19.6$)$ & 0.49 & $22.6(-17.7$ to 62.8$)$ & 0.27 \\
\hline \multicolumn{9}{|c|}{ Body mass index: } \\
\hline Week 12 & $-0.1(-0.4$ to 0.2$)$ & 0.68 & $0.1(-0.4$ to 0.5$)$ & 0.78 & $-0.1(-0.5$ to 0.4$)$ & 0.81 & $-0.2(-0.8$ to 0.4$)$ & 0.80 \\
\hline Week 24 & $0.0(-0.4$ to 0.3$)$ & 0.89 & $-0.1(-0.6$ to 0.5$)$ & 0.82 & $0.1(-0.4$ to 0.6$)$ & 0.78 & $-0.2(-0.9$ to 0.6$)$ & 0.93 \\
\hline Week 52 & $-0.4(-1.0$ to 0.2$)$ & 0.25 & $-0.2(-1.0$ to 0.7$)$ & 0.69 & $0.1(-0.7$ to 1.0$)$ & 0.79 & $-0.8(-2.2$ to 0.5$)$ & 0.23 \\
\hline \multicolumn{9}{|c|}{ Table 3 | Between group differences in primary and secondary outcomes at all weeks ${ }^{\star}$ (Continued) } \\
\hline \multicolumn{9}{|c|}{$\begin{array}{l}\text { Improved } \mathrm{HAQ} \text { score } \\
\text { (range } 0-100)+:\end{array}$} \\
\hline Week 12 & $2.1(-1.1$ to 5.2$)$ & 0.20 & $-2.2(-6.6$ to 2.2$)$ & 0.33 & $6.6(2.2$ to 11.0$)$ & 0.004 & $4.5(-1.5$ to 10.5$)$ & 0.14 \\
\hline Week 24 & $-0.1(-3.7$ to 3.5$)$ & 0.94 & $2.2(-2.9$ to 7.3$)$ & 0.39 & $1.8(-3.4$ to 6.9$)$ & 0.50 & $2.4(-4.3$ to 9.0$)$ & 0.48 \\
\hline Week 52 & $1.2(-2.7$ to 5.0$)$ & 0.54 & $-2.0(-7.6$ to 3.5$)$ & 0.47 & $4.0(-1.6$ to 9.5$)$ & 0.16 & $1.8(-5.9$ to 9.4$)$ & 0.65 \\
\hline \multicolumn{9}{|c|}{$\begin{array}{l}\text { Outcome expectations } \\
\text { scale score (range } \\
1-5) \neq:\end{array}$} \\
\hline Week 12 & $0.1(-0.1$ to 0.3$)$ & 0.48 & $0.1(-0.2$ to 0.5$)$ & 0.36 & $0.0(-0.3$ to 0.4$)$ & 0.77 & $0.3(-0.1$ to 0.7$)$ & 0.16 \\
\hline Week 24 & $0.1(-0.1$ to 0.3$)$ & 0.38 & $0.2(-0.1$ to 0.5$)$ & 0.15 & $0.1(-0.2$ to 0.4$)$ & 0.59 & $0.2(-0.1$ to 0.6$)$ & 0.20 \\
\hline Week 52 & $0.1(-0.1$ to 0.3$)$ & 0.30 & $0.1(-0.2$ to 0.4$)$ & 0.63 & $0.1(-0.2$ to 0.4$)$ & 0.46 & $0.2(-0.1$ to 0.6$)$ & 0.20 \\
\hline \multicolumn{9}{|c|}{$\begin{array}{l}\text { FIQR=revised fibromyalgia impact questionnaire; } \mathrm{HADS}=\text { hospital anxiety and depression scale; } \mathrm{MOS}=\text { Medical Outcome Study; } 36=\text { short form } 36 \text { questionnaire; } \mathrm{HAQ}=\text { health assessment } \\
\text { questionnaire. } \\
\text { P values were calculated with repeated measures analysis. } \\
\text { *All values are estimated means, with } 95 \% \text { confidence intervals. Positive scores indicate improved outcome in second listed group. Boldface indicates statistically significant differences between } \\
\text { groups. } \\
\text { tHigher scores reflect more severe symptoms. } \\
\text { fHigher scores indicate better status. }\end{array}$} \\
\hline
\end{tabular}

patients with associated comorbidities, poor quality of life, and heavy medication use. Such patients typically face limited options owing to the ineffectiveness of and contraindications to available treatments. Therefore, the overall positive findings among adults who were representative of those who are difficult to treat in routine practice strengthen the evidence that the effectiveness and durability of both tai chi and aerobic exercise extend to a diversity of patients with fibromyalgia.

We directly compared the effectiveness of two non-drug therapies that are each known to have health benefits for fibromyalgia. The novelty of the design was in the implementation of tai chi in two dosages and at two different durations, presenting an advantage over short term interventions tested in previous studies. The aerobic exercise program implemented in this trial was rigorously monitored and successfully reached its goal of intended intensity (average training heart rate ranged between 67.9\% and $79.5 \%$ of estimated maximum heart rate). This moderate intensity, together with the corresponding rate of perceived exertion, has been shown to be effective for multidimensional function, physical function, and pain ${ }^{34}$ and was also consistent across the aerobic exercise groups over the six cycles of the study. Anticipating the future generalizability of the tai chi program, we found that the therapeutic benefits were consistent for each of the three tai chi instructors. Thus, the classic Yang style tai chi can be deployed in other settings in a standardized manner for fibromyalgia. This novel design, together with the systematic enrollment efforts of this study, might enhance the generalizability of our results.

We further expect that the mind-body therapy might in the future help to reduce the burden associated with long term opioid use for patients with fibromyalgia.

The study does, however, have several limitations. First, participants were aware of their treatment group assignment, and high expectation of treatment benefit, including a placebo or nocebo response could have influenced assessments of effects. Conducting a true double blind trial design remains challenging in the absence of well accepted and valid sham comparison groups for such complex multicomponent behavioral interventions. None the less, in an attempt to mitigate the influence of pre-existing beliefs and expectations, we explicitly informed potential participants that the study was designed to test the effects of two different types of exercise programs. By emphasizing equipoise and not mentioning tai chi specifically, we hoped to decrease expectations and minimize bias. In addition, using the outcome expectations for an exercise scale to assess the possibility of bias, ${ }^{53}$ we found that participants' expectations of benefit from their randomly assigned treatment regimen were similar during baseline and follow-ups. Second, patients missed many classes and attendance differed between the two treatment groups. However, treatment effects were robust to statistical adjustments for attendance. Moreover, attendance can be considered part of the intervention, and so part of the benefit of tai chi is that patients are more likely to continue to practice 
it. Third, there was substantial loss to follow-up of participants over the course of the study both during and after the interventions. This is a common problem in clinical trials of participants with chronic pain for reasons that may be attributable to the inherent nature of their disorder. ${ }^{9}{ }^{12}$ In this study, loss to follow-up was highly correlated with attendance, because those who had poor attendance were also much more likely to fail to return for follow-up visits. Loss to follow-up was also higher among participants who had worse previous outcomes and was also correlated with several other factors. We adjusted for this missing data using a longitudinal model that incorporated all four times and conditioning on covariates related to missingness. Our results were generally robust with multiple imputation, although some estimates were slightly attenuated. Fourth, tai chi may be difficult to implement as a treatment for chronic pain in many settings for several reasons. Few programs specific to chronic pain are available in a community setting; effective practice requires instructors trained to work with a physically impaired population; as a Chinese martial art, tai chi is unfamiliar to many potential users; and effective implementation takes extensive instruction and practice. We also recognize the limited generalizability of a trial conducted in a single academic center. However, the successful dissemination of our tai chi protocol showed consistency of effects across instructors, and benefits observed in a diverse group of patients bolster potential for standardized deployment of tai chi in other settings by other instructors. Future multicenter pragmatic trials in community settings and different countries are warranted to assess the external validity of the present study and to better inform clinical practice.

One finding that deserves further investigation is that both groups in this trial showed similar reduced use of analgesics over time. It is well documented that psychosocial variables play a pivotal role in the experience of pain and aberrant opioid taking behaviors. $^{66}$ This may suggest that tai chi, which can address both the physical and the psychological symptoms in chronic pain, could be particularly effective in targeting opioid use and misuse. Future investigation of mind-body approaches should also focus on how these therapies can help reduce the burden associated with long term use of opioid analgesia.

\section{Conclusions}

Compared with aerobic exercise, the most commonly prescribed non-drug treatment, tai chi appears as effective as or better for managing fibromyalgia. Our investigation also showed that a longer duration of tai chi results in greater benefits, and patients are more likely to attend tai chi classes. The therapeutic benefits were consistent among three instructors in a large sample of diverse patients with fibromyalgia. Therefore, this mind-body approach may be considered a therapeutic option in the multidisciplinary management of fibromyalgia.
This study was overseen by an independent data and safety monitoring board and approved by the Tufts Health Sciences Campus institutional review board and the National Institutes of Health. We thank the data safety monitoring board members: Yvonne Lee, Kristin Bake, and Yulei He for their insightful suggestions concerning the study protocol; Don L Goldenberg, Egilius LH Spierings, Tammy Scott, Emily Seery, Anna Schmid, Emily Wolcott, Veronika Testa, Jolanta Marszalek, Sun Yu, Janeth Yinh, Stephanie Hyon, and Michelle Park, the Clinical and Translational Research Center nurses for their help with various aspects of the study; Ramel Rones, Dorri Li and Brian Muccio for their expertise in teaching the tai chi groups; Kimberly Martin, Eleni Tsiroyannis, Erica McDavitt, Julie Krol, Rachel Felson, and Ling Chu for their expertise in teaching the aerobic exercise group; and the study participants, whose cooperation, encouragement, and enthusiasm were an inspiration to the investigators.

Contributors: CW had full access to all of the data in the study and takes responsibility for the integrity of the data and the accuracy of the data analysis. CW, CHS, and WFH act as guarantors of this study. CW, CHS, RAF, and TMCA designed the study. CW, CHS, RAF, WFH, KFR, LLP, JBD, and RK acquired the data. All authors analyzed and interpreted the data and prepared the manuscript. CHS and LLP carried out the statistical analysis. CW obtained funding. All authors had full access to all of the data (including statistical reports and tables) in the study and can take responsibility for the integrity of the data and the accuracy of the data analysis.

Funding: This study was supported by the National Center for Complementary and Integrative Health of the National Institutes of Health (NIH, R01AT006367 and K24AT007323), the National Center for Research Resources, NIH (UL1 RR025752) and the National Center for Advancing Translational Sciences, NIH (UL1TR000073 and UL1TR001064). RAF and KRF are supported in part by supported by the US Department of Agriculture, under agreement No 58-1950-4003 and the Boston Claude D Pepper Older Americans Independence Center (1P30AG031679). The contents of this manuscript are solely the responsibility of the authors and do not necessarily represent the official views of the National Center for Complementary and Integrative Health of the National Institutes of Health. The organizations mentioned here did not have any role in the design and conduct of the study; collection, management, analysis, and interpretation of the data; preparation, review, or approval of the manuscript; or decision to submit the manuscript for publication.

Competing interests: All authors have completed the ICMJE uniform disclosure form at www.icmje.org/coi_disclosure.pdf and declare: all authors had financial support from National Center for Complementary and Integrative Health at the National Institutes of Health in the US for the submitted work; no financial relationships with any organizations that might have an interest in the submitted work in the previous three years; no other relationships or activities that could appear to have influenced the submitted work.

Ethical approval: This study was and approved by the Tufts Health Sciences Campus institutional review board and the National Institutes of Health, and informed consent was given by all patients. Data sharing: The investigators will share data (with associated coding library) used in developing the results presented in this manuscript on request to the corresponding author at cwang2@ tuftsmedicalcenter.org. Anonymized record level data will be made available on proposal for analysis by those who have received ethical clearance from their host institution.

Transparency: The lead author (CW) affirms that the manuscript is an honest, accurate, and transparent account of the study being reported; that no important aspects of the study have been omitted; and that any discrepancies from the study as planned (and, if relevant, registered) have been explained.

This is an Open Access article distributed in accordance with the Creative Commons Attribution Non Commercial (CC BY-NC 4.0) license, which permits others to distribute, remix, adapt, build upon this work non-commercially, and license their derivative works on different terms, provided the original work is properly cited and the use is noncommercial. See: http://creativecommons.org/licenses/by-nc/4.0/.

1 Wolfe F, Anderson J, Harkness D, et al. A prospective, longitudinal, multicenter study of service utilization and costs in fibromyalgia. Arthritis Rheum 1997;40:1560-70. 10.1002/art.1780400904

2 Wolfe F, Ross K, Anderson J, Russell IJ, Hebert L. The prevalence and characteristics of fibromyalgia in the general population. Arthritis Rheum 1995:38:19-28. 10.1002/art.1780380104

3 Phillips K, Clauw DJ. Central pain mechanisms in chronic pain states--maybe it is all in their head. Best Pract Res Clin Rheumatol 2011;25:141-54. 10.1016/j.berh.2011.02.005 
4 Peng X, Robinson RL, Mease P, et al. Long-term evaluation of opioid treatment in fibromyalgia. Clin J Pain 2015;31:7-13. 10.1097/ AJP.0000000000000079

5 Fitzcharles M-A, Ste-Marie PA, Gamsa A, Ware MA, Shir Y. Opioid use, misuse, and abuse in patients labeled as fibromyalgia. Am J Med 2011;124:955-60. 10.1016/j.amjmed.2011.05.031

6 Painter JT, Crofford LJ. Chronic opioid use in fibromyalgia syndrome: a clinical review. J Clin Rheumatol 2013;19:72-7. 10.1097/ RHU.0b013e3182863447

7 Goldenberg DL, Clauw DJ, Palmer RE, Clair AG. Opioid Use in Fibromyalgia: A Cautionary Tale. Mayo Clin Proc 2016;91:640-8. 10.1016/j.mayocp.2016.02.002

8 Painter JT, Crofford LJ, Talbert J. Geographic variation of chronic opioid use in fibromyalgia. Clin Ther 2013;35:303-11. 10.1016/j. clinthera.2013.02.003

9 Goldenberg D. Chronic Widespread Pain: Lessons Learned from Fibromyalgia and Related Disorders. 1st ed. Vertical Health, LLC, 2016.

10 Rooks DS, Gautam S, Romeling M, et al. Group exercise, education, and combination self-management in women with fibromyalgia: a randomized trial. Arch Intern Med 2007;167:2192-200. 10.1001/ archinte.167.20.2192

11 Busch AJ, Schachter CL, Overend TJ, Peloso PM, Barber KAR. Exercise for fibromyalgia: a systematic review. J Rheumatol 2008:35:1130-44.

12 Jones KD, Liptan GL. Exercise interventions in fibromyalgia: clinical applications from the evidence. Rheum Dis Clin North Am 2009;35:373-91. 10.1016/j.rdc.2009.05.004

13 Ramel J, Bannuru R, Griffith M, Wang C. Exercise for Fibromyalgia Pain: A Meta-Analysis of Randomized Controlled Trials. Curr Rheumatol Rev 2009;5:188-9310.2174/157339709790192459.

14 Häuser W, Klose P, Langhorst J, et al. Efficacy of different types of aerobic exercise in fibromyalgia syndrome: a systematic review and meta-analysis of randomised controlled trials. Arthritis Res Ther 2010;12:R79. 10.1186/ar3002

15 Busch AJ, Webber SC, Brachaniec M, et al. Exercise therapy for fibromyalgia. Curr Pain Headache Rep 2011;15:358-67. 10.1007/ s11916-011-0214-2

16 Bidonde J, Busch AJ, Schachter CL, et al. Aerobic exercise training for adults with fibromyalgia. Cochrane Database Syst Rev 2017;6:CD012700. 10.1002/14651858.CD012700.

17 Carville SF Arendt-Nielsen L, Bliddal $H$, et al, EULAR. EULAR evidence-based recommendations for the management of fibromyalgia syndrome. Ann Rheum Dis 2008;67:536-41. 10.1136/ ard.2007.071522

18 Buckhardt C, Goldenberg D, Crofford L, et al. Guideline for the management of fibromyalgia syndrome pain in adults and children (Clinical practice guideline; no.4). Am Pain Soc, 2005.

19 Goldenberg D. Clinical management of fibromyalgia. 1st ed. Professional Communications Inc, 2009

20 Wayne PM, Kaptchuk TJ. Challenges inherent to t'ai chi research: part I--t'ai chi as a complex multicomponent intervention. I Altern Complement Med 2008;14:95-102. 10.1089/acm.2007.7170A

21 Lauche R, Cramer H, Häuser W, Dobos G, Langhorst J. A Systematic Overview of Reviews for Complementary and Alternative Therapies in the Treatment of the Fibromyalgia Syndrome. Evid Based Complement Alternat Med 2015;2015:610615. $10.1155 / 2015 / 610615$

22 Wang C, Schmid CH, Rones R, et al. A randomized trial of tai chi for fibromyalgia. N Engl J Med 2010;363:743-54. 10.1056/ NEJMoa0912611

23 Jones KD, Sherman CA, Mist SD, Carson JW, Bennett RM, Li F. A randomized controlled trial of 8 -form Tai chi improves symptoms and functional mobility in fibromyalgia patients. Clin Rheumatol 2012:31:1205-14.10.1007/s10067-012-1996-2

24 Wang C, Schmid CH, Iversen MD, et al. Comparative effectiveness of Tai Chi versus physical therapy for knee osteoarthritis: A randomized trial Ann Intern Med 2016:165:77-86. 10.7326/M15-2143

25 Wang C. Tai Chi improves pain and functional status in adults with rheumatoid arthritis: results of a pilot single-blinded randomized controlled trial. Med Sport Sci 2008;52:218-29. $10.1159 / 000134302$

26 Lauche R, Langhorst J, Dobos G, Cramer H. A systematic review and meta-analysis of Tai Chi for osteoarthritis of the knee. Complement Ther Med 2013:21:396-406. 10.1016/j.ctim.2013.06.001

27 Lee MS, Pittler MH, Ernst E. Tai chi for osteoarthritis: a systematic review. Clin Rheumatol 2008;27:211-8. 10.1007/s10067-0070700-4

28 Burckhardt CS, Clark SR, Bennett RM. The fibromyalgia impact questionnaire: development and validation. $J$ Rheumatol 1991;18:728-33.

29 Wang C, McAlindon T, Fielding RA, et al. A novel comparative effectiveness study of Tai Chi versus aerobic exercise for fibromyalgia: study protocol for a randomized controlled trial. Trials 2015;16:34. $10.1186 / \mathrm{s} 13063-015-0548-x$
30 Wolfe F, Smythe HA, Yunus MB, et al. The American College of Rheumatology 1990 Criteria for the Classification of Fibromyalgia. Report of the Multicenter Criteria Committee. Arthritis Rheum 1990;33:160-72. 10.1002/art.1780330203

31 Wolfe F, Clauw DJ, Fitzcharles MA. The American College of Rheumatology preliminary diagnostic criteria for fibromyalgia and measurement of symptom severity. Arthritis Care Res (Hoboken) 2010;62:600-10. 10.1002/acr.20140

32 Folstein MF, Folstein SE, McHugh PR. "Mini-mental state". A practical method for grading the cognitive state of patients for the clinician. J Psychiatr Res 1975;12:189-98. 10.1016/0022-3956(75)90026-6

33 Wang C, Collet IP Lau J. The effect of Tai Chi on health outcomes in patients with chronic conditions: a systematic review. Arch Intern Med 2004;164:493-501. 10.1001/archinte.164.5.493

34 Clark M, Lucett S, Corn R. NASM essentials of personal fitness training. 3rd ed. Wolters Kluwer Health/Lippincott Williams \& Wilkins: Philadelphia, 2008.

35 Borg G. Perceived exertion as an indicator of somatic stress. Scand / Rehabil Med 1970:2:92-8.

36 Lindell L, Bergman S, Petersson IF, Jacobsson LT, Herrström P. Prevalence of fibromyalgia and chronic widespread pain. Scand J Prim Health Care 2000;18:149-53. 10.1080/028134300453340

37 NIAMS. Fibromyalgia Research Challenges and Opportunities: NIAMS. 1999. www.rightdiagnosis.com/artic/fibromyalgia_ research challenges and opportunities_niams.htm (accessed 14 Nov 2017).

38 Goldenberg DL, Burckhardt C, Crofford L. Management of fibromyalgia syndrome. JAMA 2004;292:2388-95. 10.1001/ jama.292.19.2388

39 Bennett RM, Friend R, Jones KD, Ward R, Han BK, Ross RL. The Revised Fibromyalgia Impact Questionnaire (FIQR): validation and psychometric properties. Arthritis Res Ther 2009;11:R120. 10.1186/ ar2783

40 Bennett RM, Bushmakin AG, Cappelleri JC, Zlateva G, Sadosky AB. Minimal clinically important difference in the fibromyalgia impact questionnaire. / Rheumatol 2009;36:1304-11. 10.3899/ jrheum.081090

41 Zigmond AS, Snaith RP. The hospital anxiety and depression scale. Acta Psychiatr Scand 1983;67:361-70. 10.1111/j.16000447.1983.tb09716.x

42 Lorig K, Chastain RL, Ung E, Shoor S, Holman HR. Development and evaluation of a scale to measure perceived self-efficacy in people with arthritis. Arthritis Rheum 1989;32:37-44. 10.1002/ anr.1780320107

43 Buysse DJ, Reynolds CF 3rd, Monk TH, Berman SR, Kupfer DI. The Pittsburgh Sleep Quality Index: a new instrument for psychiatric practice and research. Psychiatry Res 1989;28:193-213. 10.1016/0165-1781(89)90047-4

44 Ware JE, Kosinski M. SF-36 Physical \& Mental Health Summary Scales: A Manual for Users of Version 1. 2nd ed. QualityMetric Incorporated, 2009

45 Beck ATT, Steer RAA, Brown GKK. Manual for the Beck Depression Inventory-II. 1996. TX Psychol Corp, 1996: 1-82.

46 Rosenstiel AK, Keefe FJ. The use of coping strategies in chronic low back pain patients: relationship to patient characteristics and current adjustment. Pain 1983;17:33-44. 10.1016/03043959(83)90125-2

47 Cohen S, Mermelstein R, Kamarck T, Hoberman HM. Measuring the functional components of social support. In: Sarason IG, Sarason BR, eds. Social Support: Theory, Research and Applications. NATO ASI Series (D: Behavioural and Social Sciences), Vol 24. Springer, Dordrecht. doi.org/10.1007/978-94-009-5115-0 5 .

48 Fries JF, Cella D, Rose M, Krishnan E, Bruce B. Progress in assessing physical function in arthritis: PROMIS short forms and computerized adaptive testing / Rheumatol 2009:36:2061-6.

49 Resnick B, Zimmerman SI, Orwig D, Furstenberg AL, Magaziner J. Outcome expectations for exercise scale: utility and psychometrics. J Gerontol B Psychol Sci Soc Sci 2000;55:S352-6. 10.1093/ geronb/55.6.S352

50 Stewart AL, Mills KM, King AC, Haskell WL, Gillis D, Ritter PL. CHAMPS physical activity questionnaire for older adults: outcomes for interventions. Med Sci Sports Exerc 2001;33:1126-41. 10.1097/00005768-200107000-00010

51 Bruce B, Fries JF. The Health Assessment Questionnaire (HAQ). Clin Exp Rheumatol 2005:23(Suppl 39):S14-8.

52 Bruce B, Fries JF. The Arthritis, Rheumatism and Aging Medical Information System (ARAMIS): still young at 30 years. Clin Exp Rheumatol 2005:23(Suppl 39):S163-7.

53 Fries JF, Krishnan E. What constitutes progress in assessing patient outcomes? J Clin Epidemiol 2009;62:779-80. 10.1016/j. jclinepi.2008.12.001

54 Fries JF, Krishnan E, Rose M, Lingala B, Bruce B. Improved responsiveness and reduced sample size requirements of PROMIS physical function scales with item response theory. Arthritis Res Ther 2011:13:R147. 10.1186/ar3461. 
55 Csuka M, McCarty DJ. Simple method for measurement of lower extremity muscle strength. Am J Med 1985;78:77-81 10.1016/0002-9343(85)90465-6

56 Suzuki T, Bean JF, Fielding RA. Muscle power of the ankle flexors predicts functional performance in community-dwelling older women. J Am Geriatr Soc 2001;49:1161-7. 10.1046/j.1532 5415.2001.49232.x

57 Bean JF, Kiely DK, Leveille SG, et al. The 6-minute walk test in mobility-limited elders: what is being measured? J Gerontol A Biol Sci Med Sci 2002;57:M751-6. 10.1093/gerona/57.11.M751

58 Guyatt GH, Sullivan MJ, Thompson PJ, et al. The 6-minute walk: a new measure of exercise capacity in patients with chronic heart failure. Can Med Assoc/ 1985;132:919-23.

59 Callahan D, Phillips E, Carabello R, Frontera WR, Fielding RA Assessment of lower extremity muscle power in functionally-limited elders. Aging Clin Exp Res 2007:19:194-9. 10.1007/BF03324689

60 King S, Wessel J, Bhambhani Y, Maikala R, Sholter D, Maksymowych W. Validity and reliability of the 6 minute walk in persons with fibromyalgia. / Rheumatol 1999:26:2233-7.

61 Fitzmaurice GM, Laird NM, Ware JH. Applied Longitudinal Analysis. 2nd ed. John Wiley \& Sons, Inc, 2011.
62 Carson JW, Carson KM, Jones KD, Bennett RM, Wright CL, Mist SD. A pilot randomized controlled trial of the Yoga of Awareness program in the management of fibromyalgia. Pain 2010;151:530-9. 10.1016/j. pain.2010.08.020

63 Khoury B, Lecomte T, Fortin G, et al. Mindfulness-based therapy: a comprehensive meta-analysis. Clin Psychol Rev 2013;33:763-71. 10.1016/j.cpr.2013.05.005

64 Porter LS, Keefe FJ, McBride CM, Pollak K, Fish L, Garst J. Perceptions of patients' self-efficacy for managing pain and lung cancer symptoms: correspondence between patients and family caregivers. Pain 2002;98:169-78. 10.1016/S0304-3959(02)00042-8

65 Shelby RA, Edmond SN, Wren AA, et al. Self-efficacy for coping with symptoms moderates the relationship between physical symptoms and well-being in breast cancer survivors taking adjuvant endocrine therapy. Support Care Cancer 2014;22:2851-9. 10.1007/s00520014-2269-1

66 George SZ, Beneciuk JM. Psychological predictors of recovery from low back pain: a prospective study. BMC Musculoskelet Disord 2015;16:49. 10.1186/s12891-015-0509-2

Supplementary information: web tables 1-6 